PROCEEDINGS OF THE

AMERICAN MATHEMATICAL SOCIETY

Volume 130, Number 4, Pages 963-969

S 0002-9939(01)06267-0

Article electronically published on October 1, 2001

\title{
INTERPRETATION OF THE DEFORMATION SPACE OF A DETERMINANTAL BARLOW SURFACE VIA SMOOTHINGS
}

\author{
YONGNAM LEE
}

(Communicated by Michael Stillman)

\begin{abstract}
In this present paper, we provide an interpretation of the deformation space of a determinantal Barlow surface via smoothings.
\end{abstract}

\section{INTRODUCTION}

Let $\left(y_{1}, y_{2}, y_{3}, y_{4}\right)$ be a coordinate of $\mathbb{P}^{3}$, and consider a $D_{5}=\langle b, a\rangle$ action on $\mathbb{P}^{3}$ via

$$
\begin{aligned}
& b:\left(y_{1}, y_{2}, y_{3}, y_{4}\right) \rightarrow\left(\epsilon y_{1}, \epsilon^{2} y_{2}, \epsilon^{3} y_{3}, \epsilon^{4} y_{4}\right), \\
& a:\left(y_{1}, y_{2}, y_{3}, y_{4}\right) \rightarrow\left(y_{4}, y_{3}, y_{2}, y_{1}\right)
\end{aligned}
$$

where $\epsilon$ is the primitive 5-th root of unity. In [C1], Catanese studied a fourdimensional family of surfaces that are double coverings of $\mathbb{Z}_{5}$-quotients of the $\mathbb{Z}_{5}$-invariant symmetric determinantal quintics. We will refer to these $\mathbb{Z}_{5}$-quotients of the $\mathbb{Z}_{5}$-invariant symmetric determinantal quintics as determinantal Godeaux surfaces. Inside of this four-dimensional family, there is a two-dimensional $D_{5^{-}}$ invariant symmetric determinantal Godeaux surface. After providing a twisted $\mathbb{Z}_{2}$-action on the double cover, we have a two-dimensional subfamily of the moduli space of Barlow's example $[\bar{B}$. We will refer to these surfaces as determinantal Barlow surfaces. Let $\Sigma$ be a $D_{5}$-invariant symmetric determinantal quintic surface in $\mathbb{P}^{3}$ and let $\sigma: Y \rightarrow \Sigma$ be a double cover. Consider a commutative diagram

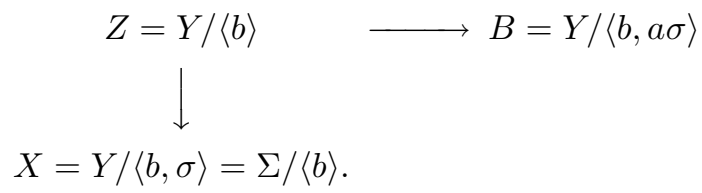

In diagram (1.1), $X$ is a $D_{5}$-invariant determinantal Godeaux surface, and $B$ is a determinantal Barlow surface. By the construction of a $D_{5}$-invariant determinantal Godeaux surface, there is an extra involution. The fixed divisor of this involution is a -3-curve $D_{X}\left(\mathbb{P}^{1}\right.$ with $\left.N_{\mathbb{P}^{1} \mid X}=\mathcal{O}_{\mathbb{P}^{1}}(-3)\right)$ without passing through four nodes (the double cover $Z \rightarrow X$ is branched over these four nodes). So the preimage of $D_{X}$ in $Z$ is two disjoint -3 -curves, $D_{1}, D_{2}$. Since $a$-action fixes each point on $D_{1}$

Received by the editors May 10, 2000 and, in revised form, October 17, 2000.

2000 Mathematics Subject Classification. Primary 14J10, 14J17.

This work was supported by grant 1999-2-102-002-3 from the Interdisciplinary Research Program of the KOSEF and by the Sogang University Research Grants in 2000.

(C)2001 American Mathematical Society 
and $D_{2}, \mathbb{Z}_{2}$-action of $Z \rightarrow B$ interchanges $D_{1}$ and $D_{2}$. So these two -3 -curves go to a -3 -curve $D_{B}$ in $B$ without passing through four nodes (the double cover $Z \rightarrow B$ is branched over these four nodes) [B]. By parameterizing $\mathbb{Z}_{5}$-invariant symmetric determinantal quintics with 20 nodes, we can easily see that the general element of those set do not have the preimage of $D_{X}$. This argument shows that the natural map of $H^{1}\left(T_{X}\right) \rightarrow H^{1}\left(N_{D_{X} \mid X}\right)$ is surjective. Therefore we have two directions of the first order deformation space induced by the cohomology of $H^{1}\left(N_{D_{X} \mid X}\right)$. In this paper, we will prove that the surjectivity of the map of $H^{1}\left(T_{X}\right) \rightarrow H^{1}\left(N_{D_{X} \mid X}\right)$ produces the surjectivity of the map of $H^{1}\left(T_{B}\right) \rightarrow H^{1}\left(N_{D_{B} \mid B}\right)$ by the lifting of the first order deformation space to the commutative diagram (1.1) and the vanishing of $H^{2}\left(T_{Z}\right)=0$. This vanishing was proved by Catanese-Le Brun [CL] and by the author $[\mathrm{L}$ independently. Then we provide an interpretation of the deformation space of a determinantal Barlow surface via smoothings :

Theorem. Consider a determinantal Barlow surface. Then the extension of the two-dimensional family of the determinantal Barlow surface, in the recovering six directions, can be interpreted as follows:

(1) one independent smoothing direction for each of the four nodes of $B$,

(2) two independent directions corresponding to the global smoothings induced by the surjectivity of the map $H^{1}\left(T_{B}\right) \rightarrow H^{1}\left(N_{D_{B} \mid B}\right)$.

Throughout we work over the complex number field $\mathbb{C}$. The notation here follows the standard textbook $[\mathrm{H}]$.

\section{Proof of the Theorem}

Let $V$ be a smooth projective surface and $D$ a smooth curve in $V$. There is a natural map $n: T_{V} \rightarrow N_{D \mid V}$ induced by the composition of the maps $T_{V} \rightarrow$ $\left.T_{V}\right|_{D} \rightarrow N_{D \mid V}$. Define $T_{V, D}$ by the kernel of the map of $n$ :

$$
0 \rightarrow T_{V, D} \rightarrow T_{V} \stackrel{n}{\rightarrow} N_{D \mid V} \rightarrow 0 .
$$

The short exact sequence (2.1) induces the long exact sequence

$$
\begin{gathered}
0 \rightarrow H^{0}\left(T_{V, D}\right) \rightarrow H^{0}\left(T_{V}\right) \rightarrow H^{0}\left(N_{D \mid V}\right) \rightarrow H^{1}\left(T_{V, D}\right) \\
\rightarrow H^{1}\left(T_{V}\right) \rightarrow H^{1}\left(N_{D \mid V}\right) \rightarrow H^{2}\left(T_{V, D}\right) \rightarrow H^{2}\left(T_{V}\right) .
\end{gathered}
$$

In the long exact sequence (2.2), each cohomology group relates the first order infinitesimal deformation or obstruction:

(2.2.1) $H^{0}\left(N_{D \mid V}\right)$ classes the first order infinitesimal deformation of $D$ in $V$ and the obstruction lies in $H^{1}\left(N_{D \mid V}\right)$,

(2.2.2) $H^{1}\left(T_{V, D}\right)$ classes the first order infinitesimal deformation of the pair $(V, D)$ and the obstruction lies in $H^{2}\left(T_{V, D}\right)$,

(2.2.3) $H^{1}\left(T_{V}\right)$ classes the first order infinitesimal deformation of $V$ and the obstruction lies in $H^{2}\left(T_{V}\right)$.

We also have the commutative diagram of the obstruction maps in (2.2):

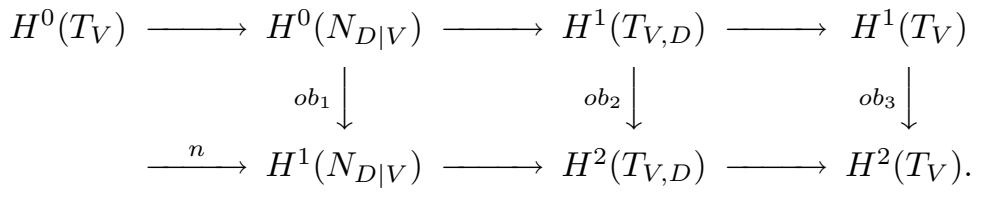

The next lemma is easily obtained by the commutative diagram (2.3). 
Lemma 1. (1) Assume that the natural map $n: H^{1}\left(T_{V}\right) \rightarrow H^{1}\left(N_{D \mid V}\right)$ is surjective. Then $o b_{3}=0$ implies that $o b_{2}=0$.

(2) If $H^{1}\left(N_{D \mid V}\right)=0$, then $o b_{2}=0$ if and only if $o b_{3}=0$.

The definition of $T_{V, D}$ induces a short exact sequence:

$$
0 \rightarrow T_{V}\left(I_{D}\right) \rightarrow T_{V, D} \stackrel{t}{\rightarrow} T_{D} \rightarrow 0 .
$$

It is interesting to find the cases when the natural maps $n: H^{1}\left(T_{V}\right) \rightarrow H^{1}\left(N_{D \mid V}\right)$ or $t: H^{1}\left(T_{V, D}\right) \rightarrow H^{1}\left(T_{D}\right)$ are zero or surjective.

Definition 2. A simple normal crossing surface $X$ (a projective surface with normal crossing, and each irreducible component is smooth) is smoothable if there is an analytic disc $\Delta$ in $\mathbb{C}$ and a projective flat family of varieties $\pi: \mathcal{X} \rightarrow \Delta$ whose central fiber is $X$, and a general fiber $X_{t}=\pi^{-1}(t)$ is smooth for $t \neq 0$. Then $\pi: \mathcal{X} \rightarrow \Delta$ is called a smoothing of $X$.

Denote by $T_{X}^{i}$ and $\mathbb{T}_{X}^{i}$ the local and global deformation objects of LichtenbaumSchlessinger, respectively. In the case of reduced local complete intersection, we have

$$
T_{X}^{i}=\mathcal{E} x t_{X}^{i}\left(\Omega_{X}, \mathcal{O}_{X}\right), \mathbb{T}_{X}^{i}=\operatorname{Ext}_{X}^{i}\left(\Omega_{X}, \mathcal{O}_{X}\right) .
$$

Since $X$ is normal crossing, $X$ is locally embedded in a smooth variety $\mathcal{X}$ and we have

$$
0 \rightarrow I_{X} /\left.I_{X}^{2} \rightarrow \Omega_{\mathcal{X}}\right|_{X} \rightarrow \Omega_{X} \rightarrow 0
$$

where $I_{X} / I_{X}^{2}$ is locally free $\mathcal{O}_{X}$-module. Therefore $T_{X}^{2}=0$.

There exists a spectral sequence $E_{2}^{p, q}=H^{p}\left(X, \mathcal{E} x t_{X}^{q}\left(\Omega_{X}, \mathcal{O}_{X}\right)\right)$ and $E_{\infty}$ goes to $\mathcal{E} x t_{X}^{p+q}\left(\Omega_{X}, \mathcal{O}_{X}\right)$. Also, there is a local-global exact sequence $(\underline{\mathrm{Go}}, \S 7.3)$

$$
\begin{aligned}
0 & \rightarrow H^{1}\left(X, T_{X}^{0}\right) \rightarrow \operatorname{Ext}_{X}^{1}\left(\Omega_{X}, \mathcal{O}_{X}\right) \rightarrow H^{0}\left(X, \mathcal{E} x t_{X}^{1}\left(\Omega_{X}, \mathcal{O}_{X}\right)\right) \\
\rightarrow H^{2}\left(X, T_{X}^{0}\right) & \rightarrow \operatorname{Ext}_{X}^{2}\left(\Omega_{X}, \mathcal{O}_{X}\right) \rightarrow H^{1}\left(X, \mathcal{E} x t_{X}^{1}\left(\Omega_{X}, \mathcal{O}_{X}\right)\right),
\end{aligned}
$$

so the following long exact sequence holds:

$$
0 \rightarrow H^{1}\left(T_{X}^{0}\right) \rightarrow \mathbb{T}_{X}^{1} \rightarrow H^{0}\left(T_{X}^{1}\right) \rightarrow H^{2}\left(T_{X}^{0}\right) \rightarrow \mathbb{T}_{X}^{2} \rightarrow H^{1}\left(T_{X}^{1}\right)
$$

The space $H^{1}\left(T_{X}^{0}\right)$ classifies all "locally trivial" deformations of $X$, i.e. for which the singularities remain locally a product, and its obstruction lies in $H^{2}\left(T_{X}^{0}\right)$. So if there is a smoothing of $X$, then $\mathbb{T}_{X}^{1} \rightarrow H^{0}\left(T_{X}^{1}\right)$ is not zero. Let $D=\operatorname{Sing} X$. If there is a smoothing $\mathcal{X}$ of $X$, then $T_{X}^{1} \cong \mathcal{O}_{D}(E)$ for some effective divisor $E$. In addition, if $\mathcal{X}$ is assumed to be smooth, then $T_{X}^{1} \cong \mathcal{O}_{D}$. In general, $T_{X}^{1}$ is not $\mathcal{O}_{D}$ [F, §2]. For example if $X=V \cup_{D} W$, then $T_{X}^{1} \cong N_{D \mid V} \otimes N_{D \mid W}$.

Definition 3. Let $X$ be a simple normal crossing variety. Assume $T_{X}^{1} \cong \mathcal{O}_{D}$ for $D=\operatorname{Sing} X$. Then $X$ is called $d$-semistable.

We consider the simplest case: $X=V \cup_{D} W$ with $T_{X}^{1} \cong \mathcal{O}_{D}$. Then there are relations between $T_{X}^{0}$, and $T_{V, D}, T_{W, D}$ by definition of $T_{X}^{0}$.

$$
\begin{aligned}
0 & \rightarrow T_{X}^{0} \rightarrow T_{V, D} \oplus T_{W, D} \stackrel{\frac{1}{2}\left(t_{1}+t_{2}\right)_{*}}{\longrightarrow} T_{D} \rightarrow 0, \\
0 & \rightarrow T_{V}(-D) \oplus T_{W}(-D) \rightarrow T_{X}^{0} \rightarrow T_{D} \rightarrow 0 .
\end{aligned}
$$


Then the map $H^{0}\left(N_{D \mid V} \otimes N_{D \mid W}\right) \rightarrow H^{2}\left(T_{V}\right) \oplus H^{2}\left(T_{W}\right)$, that is a composition of the map $H^{0}\left(N_{D \mid V} \otimes N_{D \mid W}\right) \rightarrow H^{2}\left(T_{X}^{0}\right)$ and the maps induced by the exact sequences (2.6), (2.1), can be obtained via the following natural maps DF].

Let $\omega_{W}$ be the obstruction class of the extension of the first-order neighborhood of $D$ in $W$ to the flat model, which is in $H^{1}\left(N_{D \mid W}^{*} \otimes T_{D}\right)$. The splitting of the exact sequence of $\left.0 \rightarrow T_{D} \rightarrow T_{W}\right|_{D} \rightarrow N_{D \mid W} \rightarrow 0$ is the same as $\omega_{W}=0$. Consider the natural map

$$
\begin{aligned}
H^{0}\left(N_{D \mid V} \otimes N_{D \mid W}\right) \otimes \omega_{W} & \rightarrow H^{1}\left(N_{D \mid V} \otimes T_{D}\right) \\
& \rightarrow H^{1}\left(\left.N_{D \mid V} \otimes T_{V}\right|_{D}\right) \\
& \rightarrow H^{2}\left(T_{V}\right)
\end{aligned}
$$

induced by the exact sequence $\left.0 \rightarrow T_{V} \rightarrow T_{V}(D) \rightarrow T_{V}(D)\right|_{D} \rightarrow 0$. The other direction is exactly the same as this. Then the next lemma is easily obtained by the observation.

Lemma 4. Assume that the exact sequence $\left.0 \rightarrow T_{D} \rightarrow T_{W}\right|_{D} \rightarrow N_{D \mid W} \rightarrow 0$ splits and that $H^{2}\left(T_{W}\right)$ vanishes. Then the map $H^{0}\left(T_{X}^{1}\right) \rightarrow H^{2}\left(T_{V}\right) \oplus H^{2}\left(T_{W}\right)$ is zero. In particular, if $W$ is a ruled surface and $D$ is a section in $W$, then the map $H^{0}\left(T_{X}^{1}\right) \rightarrow H^{2}\left(T_{V}\right) \oplus H^{2}\left(T_{W}\right)$ is zero.

The next example in $[\mathrm{PP}$ shows that the vanishing of the above map is not enough for the obstruction map to be zero even for a $d$-semistable case.

Example 5. Let $V$ be a smooth projective surface and $D$ a smooth curve in $V$. Choose an element $\eta \in H^{1}\left(N_{D \mid V}\right)$. Since $H^{1}\left(N_{D \mid V}\right)=\operatorname{Ext}^{1}\left(\mathcal{O}_{D}, N_{D \mid V}\right), \eta$ corresponds to a vector bundle $\mathcal{E}$ of rank two over $D$ by the extension

$$
0 \rightarrow N_{D \mid V} \rightarrow \mathcal{E} \rightarrow \mathcal{O}_{D} \rightarrow 0 .
$$

Let $W=\mathbb{P}_{D}(\mathcal{E})$ and let $X=V \cup_{D} W$. Then $X$ satisfies $d$-semistability by its construction. In $[\mathrm{PP}, \S 2]$, Persson and Pinkham prove that the image of the map $H^{0}\left(T_{X}^{1}\right) \rightarrow H^{2}\left(T_{V, D}\right)$, induced by the composition of the maps $H^{0}\left(T_{X}^{1}\right) \rightarrow$ $H^{2}\left(T_{X}^{0}\right) \rightarrow H^{2}\left(T_{V, D}\right)+H^{2}\left(T_{W, D}\right) \rightarrow H^{2}\left(T_{V, D}\right)$ where the last map is the projection, is same as the image of $\eta$ in $H^{2}\left(T_{V, D}\right)$ induced by the exact sequence (2.1). Therefore if $\eta$ is not in the image of the map $n: H^{1}\left(T_{V}\right) \rightarrow H^{1}\left(N_{D \mid V}\right)$, then there is no smoothing of $X$.

Lemma 6. In Example 5, if we assume that the map $H^{1}\left(T_{W}\right) \rightarrow H^{1}\left(N_{D \mid W}\right)$ is surjective, then the obstructiom map $H^{0}\left(T_{X}^{1}\right) \rightarrow H^{2}\left(T_{X}^{0}\right)$ is zero if and only if $\eta$ is the image of $n$ for $\eta \in H^{1}\left(N_{D \mid V}\right)$.

Proof. Since $W$ is a ruled surface, we have the vanishing $H^{2}\left(T_{W}\right)=0$. Then the assumption implies that $H^{2}\left(T_{W, D}\right)=0$ by the exact sequence (2.1). Since $W$ is a ruled surface, the map $H^{1}\left(T_{W}\right) \rightarrow H^{1}\left(T_{D}\right)$, induced by the splitting of the exact sequence (2.1), is surjective. This splitting also applies to the surjectivity of the map $t: H^{1}\left(T_{W, D}\right) \rightarrow H^{1}\left(T_{D}\right)$ induced by the exact sequence (2.4). Therefore $H^{2}\left(T_{X}^{0}\right)=H^{2}\left(T_{V, D}\right)$ by the exact sequence (2.6).

Lemma 7. In Example 5, if we assume that $H^{2}\left(T_{V}\right)=0$, then $X$ has a smoothing if and only if $\eta$ is the image of $n$. 
Proof. If $X$ has a smoothing, then the map $H^{0}\left(T_{X}^{1}\right) \rightarrow H^{2}\left(T_{X}^{0}\right)$ is zero. So $\eta$ is in the image of $n$ by the argument in Example 5. Assume that $\eta$ is in the image of $n$. So there is an element $\theta$ in $H^{1}\left(T_{V}\right)$ such that $n(\theta)=\eta$. Since $H^{2}\left(T_{V}\right)=0$, we have the $\theta$-direction deformation of $V$ i.e. there is a flat family $\pi: \mathcal{V} \rightarrow \Delta$ with $\pi^{-1}(0)=V$ and $\theta$ corresponds to the extension $\left.0 \rightarrow T_{V} \rightarrow T_{\mathcal{V}}\right|_{V} \rightarrow \mathcal{O}_{V} \rightarrow 0$. Blow up $D$ in $\mathcal{V}$; then we have a smoothing of $X$.

Example 8. Let $V_{1}, W_{1}$ be smooth projective surfaces. Assume that $\sigma: V_{1} \rightarrow W_{1}$ is a double covering branched over a finite disjoint union of irreducible smooth curves. Assume that there is a smooth curve $D$ in $W_{1}$ outside of branch loci. Write $\sigma^{-1}(D)=D_{1}+D_{2}, D \cong D_{i}$ for $i=1,2$. Then the analytic neighborhood of $D$ in $W_{1}$ is isomorphic to that of $D_{1}$ in $V_{1}$, in particular $N_{D \mid V_{1}} \cong N_{D \mid W_{1}}$. For convenience we also write $D_{1}=D$.

Choose a nonzero element $\eta$ in $H^{1}\left(N_{D \mid V_{1}}\right)=H^{1}\left(N_{D \mid W_{1}}\right)$. Construct $W_{2}=$ $\mathbb{P}_{D}(\mathcal{E})$ where $\mathcal{E}$ corresponds to the extension sheaf of $\eta$ (Example 5). Let $X=$ $V_{1} \cup_{D} W_{2}$ and $Y=W_{1} \cup_{D} W_{2}$. Then one may guess the following statement: $X$ has a smoothing if and only if $Y$ has a smoothing. But this is not true in general by the next example.

Example 9. Choose a smooth curve $C$ with $g(C) \geq 2$ and a finite morphism $f: C \rightarrow \mathbb{P}^{1}$. This induces a finite morphism $\hat{f}$ and a commutative diagram

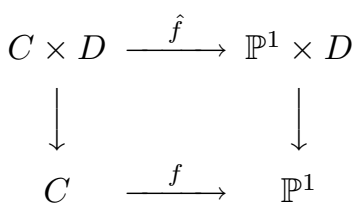

where $D$ is a smooth curve with $g(D) \geq 2$. Then $\hat{f}$ is branched over finite fibers $D_{1}, \ldots, D_{n}$. Choose a fiber $D_{0}$ such that $D_{0}$ is outside of $\left\{D_{1}, \ldots, D_{n}\right\}$. Let $V_{1}=C \times D, W_{1}=\mathbb{P}^{1} \times D$. Since we choose $D_{0}$ outside of the branch loci,

$$
N_{D_{0} \mid V_{1}} \cong N_{D_{0} \mid W_{1}} \cong \mathcal{O}_{D_{0}} \text {. }
$$

Choose a nonzero element $\eta \in H^{1}\left(\mathcal{O}_{D_{0}}\right)$. Construct $W_{2}=\mathbb{P}_{D_{0}}(\mathcal{E})$. Then $X=$ $V_{1} \cup_{D_{0}} W_{2}$ has no smoothing by Lemma 10 and Example 5 , but $Y=W_{1} \cup_{D_{0}} W_{2}$ has a smoothing by Lemma 11 .

Lemma 10. Let $C, D$ be smooth curves of genus $g \geq 2$, and let $X=C \times D$. Then the map $n: H^{1}\left(T_{X}\right) \rightarrow H^{1}\left(N_{C \mid X}\right)$ is zero.

Proof. It holds that $H^{1}\left(T_{X}\right)=H^{1}\left(T_{C}\right) \oplus H^{1}\left(T_{D}\right)$. The map $n$ restricted on $H^{1}\left(T_{C}\right)$ to $H^{1}\left(N_{C \mid X}\right)$ is derived from the splitting of the long exact sequence of $0 \rightarrow T_{C} \rightarrow$ $\left.T_{X}\right|_{C} \rightarrow N_{C \mid X} \rightarrow 0$. Also, the map $n$ restricted on $H^{1}\left(T_{D}\right)$ to $H^{1}\left(N_{C \mid X}\right)$ is zero since $D$ lies on the fiber of $X \rightarrow C$.

Lemma 11. In Example 9, $Y=W_{1} \cup_{D_{0}} W_{2}$ has a smoothing. And the natural map $n: H^{1}\left(T_{W_{1}}\right) \rightarrow H^{1}\left(N_{D_{0} \mid W_{1}}\right)$ is surjective.

Proof. Choose any $\eta \in H^{1}\left(N_{D_{0} \mid W_{1}}\right)=H^{1}\left(\mathcal{O}_{D_{0}}\right)$. Consider a trivial family $\mathcal{W}$ of $W_{2}=\mathbb{P}_{D_{0}}(\mathcal{E})$ where $\mathcal{E}$ corresponds to an extension sheaf of $\eta$. Blow up the central fiber; then we have $Y=W_{1} \cup_{D_{0}} W_{2}$ in the central fiber. So $Y$ has a smoothing. By Lemma 7, $\eta$ is in the image of $n$. 
According to the above example, the same analytic neighborhood of the double curve is not enough to determine whether a smoothing exists or not. Let us go back to the question in Example 8. Let $\sigma: V_{1} \rightarrow W_{1}$ be a double covering branched over $C$ where $C$ consists of a finite disjoint union of smooth curves. There is a relation between the cohomology of the tangent sheaf of $V_{1}$ and the cohomology of the tangent sheaf of $W_{1}[\mathrm{C} 2$ :

$$
0 \rightarrow \sigma_{*}\left(\sigma^{*} \Omega_{W_{1}}\left(K_{V_{1}}\right)\right) \rightarrow \sigma_{*}\left(\Omega_{V_{1}}\left(K_{V_{1}}\right)\right) \rightarrow \mathcal{O}_{C}\left(K_{W_{1}}\right) \rightarrow 0
$$

and it holds the long exact sequence by Serre duality

$$
0 \rightarrow H^{0}\left(T_{V_{1}}\right) \rightarrow H^{0}\left(T_{W_{1}}\right) \oplus H^{0}\left(T_{W_{1}}(-L)\right) \rightarrow H^{0}\left(\mathcal{O}_{C}(C)\right) \rightarrow H^{1}\left(T_{V_{1}}\right) \rightarrow \ldots
$$

where $2 L \sim C$. In particular, $H^{2}\left(T_{V_{1}}\right)=0$ implies that $H^{2}\left(T_{W_{1}}\right)=0$.

Proposition 12. Consider Example 8 under the assumption $H^{2}\left(T_{V_{1}}\right)=0$. Let $C$ be a branched divisor. Assume that $D=\mathbb{P}^{1}$ or $L$ itself is effective for $2 L \sim C$. Then we have the following:

(1) If $X$ has a smoothing, then $Y$ has a smoothing.

(2) If $Y$ has a smoothing and the preimage of $\eta$ in $H^{1}\left(T_{W_{1}}\right)$ goes to zero in $H^{1}\left(N_{C \mid W_{1}}\right)$, then $X$ has a smoothing.

Proof. Since we have the vanishing $H^{2}\left(T_{V_{1}}\right)=H^{2}\left(T_{W_{1}}\right)=0$, the smoothability of $X$ and $Y$ is determined by the image of the following natural maps by Lemma 7 :

$$
n_{1}: H^{1}\left(T_{V_{1}}\right) \rightarrow H^{1}\left(N_{D \mid V_{1}}\right) n_{2}: H^{1}\left(T_{W_{1}}\right) \rightarrow H^{1}\left(N_{D \mid W_{1}}\right) .
$$

Since $D=\mathbb{P}^{1}$ or $L$ is effective and since $D$ is outside of the branched divisor $C$, we have $\sigma_{*} \sigma^{*} N_{D \mid W_{1}}(-L)=N_{D \mid W_{1}}$.

From the commutative diagram (in the diagram, the vertical map is induced by the dual sequence of the exact sequence (2.8))

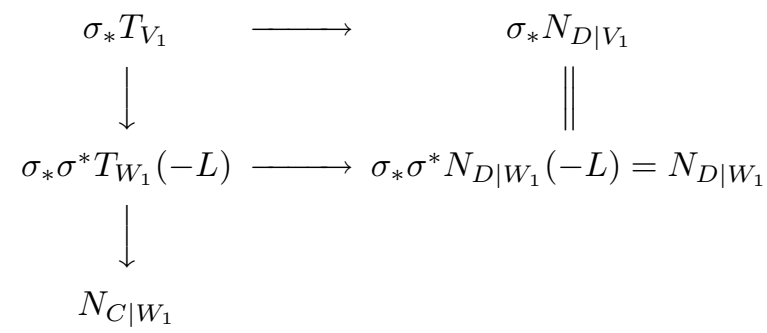

the following commutative diagram in the long exact sequence of cohomology holds:

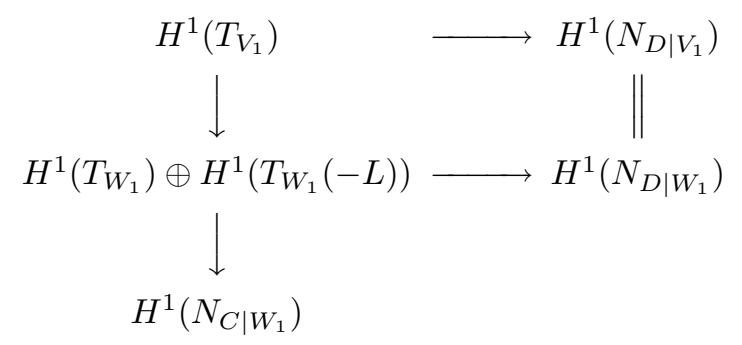

By the splitting of the eigenspaces of $\mathbb{Z}_{2}$-action, the maps of $H^{1}\left(T_{W_{1}}(-L)\right) \rightarrow$ $H^{1}\left(N_{D \mid W_{1}}\right)$ and of $H^{1}\left(T_{W_{1}}(-L)\right) \rightarrow H^{1}\left(N_{C \mid W_{1}}\right)$ are zero. Then the proof is obtained by the commutative diagram (2.10) and Lemma 7.

By Proposition 12, we finish the proof of the Theorem. 


\section{REFERENCES}

[B] R. Barlow, A simply connected surface of general type with $p_{g}=0$, Invent. Math. 79 (1985), 293-301. MR 87a:14033

[C1] F. Catanese, Babbage's conjecture, contact of surfaces, symmetric determinantal varieties and applications, Invent. Math. 63 (1981), 433-465. MR 83c:14026

[C2] F. Catanese, On the moduli spaces of surfaces of general type, J. Diff. Geom. 19 (1984), 483-515. MR 86h:14031

[CL] F. Catanese and C. Le Brun, On the scalar curvature of Einstein manifolds, Math. Res. Lett. 4, No. 6 (1997), 843-854. MR 98k:53057

[DF] S. Donaldson and R. Friedman, Connected sums of self-dual manifolds and deformations of singular spaces, Nonlinearity 2 (1989), 197-239. MR 90e:32027

[F] R. Friedman, Global smoothings of varieties with normal crossings, Ann. Math. 118 (1983), 75-114. MR 85g:32029

[Go] R. Godement, Topologie Algébrique et Théorie des Faisceau, Hermann, Paris, 1958. MR 49:9831

[H] R. Hartshorne, Algebraic Geometry, Graduate Texts in Math., vol. 52, Springer-Verlag, 1977. MR 57:3116

[L] Y. Lee, Bicanonical pencil of a determinantal Barlow surface, Trans. Amer. Math. Soc. 353 (2001), 893-905. MR 2001f:14075

[PP] U. Persson and H. Pinkham, Some examples of nonsmoothable varieties with normal crossings, Duke Math. J. 50, No. 2 (1983), 477-486. MR 84k:14010

Department of Mathematics, Sogang University, Sinsu-dong, Mapo-gu, Seoul 121742 , KOREA

E-mail address: ynlee@ccs.sogang.ac.kr 